\title{
PEDF promotes retinal neurosphere formation and expansion in vitro
}

\author{
Anna De Marzo, Claudia Aruta and Valeria Marigo. \\ Department of Biomedical Sciences, University of Modena and Reggio Emilia, Modena, Italy
}

\section{Introduction}

Many forms of blindness arise from photoreceptor degeneration and to date have no satisfactory solutions to rescue retinal tissues.

Mammalian eyes do not have a regenerative capability characteristic of lower vertebrates but recent evidences have demonstrated that ciliary body (CB), a structure analogous to the $\mathrm{CMZ}$ of lower vertebrates, contains retinal stem cells (RSC) (Tropepe et al., 2000; Ahmad et al., 2000; Coles et al., 2004; Inoue et al., 2005).

Multipotent retinal stem or progenitor cells can be isolated from the ciliary body of an adult mammalian retina using a neurosphere culture. Tropepe et al., have demonstrated that one out of $500 \mathrm{CB}$ cells gives rise to a clonal aggregate called neurosphere consisting of $\sim 12000$ pigmented cells and that each neurosphere can generate six to eight daughter neurosphere colonies.

Although the presence and the function of retinal stem cells (RSCs) in vivo remains unknown RSCs proliferate in vitro; they differentiate into cell subtypes expressing markers of certain mature retinal neurons such as bipolar cells, photoreceptor cells or Muller glia suggesting the possibility that these cells may represent a potential cell source in transplantation therapy for retinal diseases (Tropepe et al., 2000, Giordano et al., 2007). Differentiation is induced by plating the cells on an extracellular matrix substrate and exposing the cells to $1 \%$ Fetal Bovine Serum (FBS). In these conditions the percentage of cells that undertake a rod-like fate is about $30-40 \%$ as assessed by rhodopsin and the Pde6b co-expression. 
Growth of neurospheres with FGF without EGF before differentiation favors rod-like differentiation (Giordano et al.).

Primary cultures of cells collected from the CB are in FBS free medium and the formation of neurospheres takes about 5-7 days. The number of neurospheres is dependent upon the growth factors to which cells are exposed, such as FGF or EGF. A limitation in the culture of RSC comes from low ability of these cells to be expanded in vitro. In fact, a single retinal neurosphere composed of about 12,000 cells upon passaging does not give rise to 12,000 new spheres but only an average of 3 . Furthermore, no expansion of the culture has been obtained from the third passage (Giordano et al). Amelioration of the culture condition is therefore a fundamental aspect that needs to be addressed if we want to bring RSC to therapeutic applications.

Previous works have shown that pigment epithelium-derived factor (PEDF) secreted by the murine subventricular zone (SVZ) promotes self-renewal and activation of slowly dividing adult neural stem cells (NSC) in vitro (Ramirez-Castillejo et al., 2006). PEDF is a neurotrophic antiangiogenic factor initially purified from conditioned media of retinal pigment epithelial cells (Tombran-Tink et al., 1991; Becerra et al., 1995). PEDF is secreted from many different cells and can modulate cell cycle progression (Pignolo et al., 2003). Thus PEDF may have positive effects on RSC culture.

Here we show that the treatment of RSC with PEDF together with FGF increases the neurosphere yield and, ameliorates self-renewal in neurosphere passaging. We then tested whether PEDF could affect differentiation to rod-like fate. This improvement does not decrease the number of cells expressing rhodopsin and Pde6b. Moreover, cells differentiated from a FGF+PEDF culture did not express bipolar cell markers.

\section{Materials and Methods}




\section{Retinal stem cell isolation and culture}

All procedures on mice (including their euthanasia) were performed in accordance with the ARVO Statement for the Use of Animals in Ophthalmic and Vision Research and with institutional guidelines for animal research. In this study we used C57BL/6 mice purchased from Charles River Italy (Calco, Italy) and housed them under standard conditions with a 12-hour light/dark cycle. We dissected eyes from 12-week-old C57BL6 mice in artificial cerebral spinal fluid (ACSF) containing $124 \mathrm{mM} \mathrm{NaCl}, 5$ $\mathrm{mM} \mathrm{KCl}, 100 \mathrm{nM} \mathrm{CaCl} 2,1.3 \mathrm{mM} \mathrm{MgCl} 2,26 \mathrm{mM} \mathrm{NaHCO} 3$ and $10 \mathrm{mM}$ D-glucose. Eyes were cut in two hemispheres and the lens and the neural retina were carefully removed. The ciliary body was separated from the retinal pigment epithelium (RPE), treated with trypsin and hyaluronidase and the ciliary margin cells were scraped from the sclera according to the procedures described in the paper (Giordano et al, 2007). The isolated cells were grown in a serum free medium ( $0.6 \%$ glucose and $\mathrm{N} 2$ hormone mix in DMEM-F12) containing either $20 \mathrm{ng} / \mathrm{ml}$ basic FGF (FGF) supplemented with $2 \mu \mathrm{g} / \mathrm{ml}$ heparin (Sigma, Milan, Italy) or both FGF $+20 \mathrm{ng} / \mathrm{ml}$ PEDF (Chemicon). Cells were seeded at a concentration of 40,000 cells $/ \mathrm{ml}$ and incubated for 6 days until floating spheres formed.

\section{Single sphere passaging}

A single sphere was placed in a microcentrifuge tube and incubated in enzyme solution (ACSF containing $1.33 \mathrm{mg} / \mathrm{ml}$ trypsin, $0.67 \mathrm{mg} / \mathrm{ml}$ hyaluronidase, $0.5 \mathrm{mg} / \mathrm{ml}$ collagenase type I-A, $0.5 \mathrm{mg} / \mathrm{ml}$ collagenase XI, $0.13 \mathrm{mg} / \mathrm{ml}$ kynurenic acid) for 1 hour at $37{ }^{\circ} \mathrm{C}$. After centrifugation at $400 \mathrm{~g}$ for $5 \mathrm{~min}$ the supernatant was replaced with $1 \mathrm{mg} / \mathrm{ml}$ of trypsin inhibitor in medium and the sphere was mechanically dissociated. Collected cells from each sphere were seeded into a 96-well plate in serumfree medium containing FGF or FGF+PEDF and incubated for six days. 


\section{Bromodeoxyuridine labeling}

Retinal floating spheres at five days of culture were treated with $10 \mathrm{mM}$ bromodeoxyuridine (BrdU) for 3 hours, washed with PBS, allowed to attach to a slide and fixed in 4\% paraformaldehyde (PFA). Spheres were treated with $2 \mathrm{~N} \mathrm{HCl}$ at $30{ }^{\circ} \mathrm{C}$ for $30 \mathrm{~min}$, placed in a $0.1 \mathrm{M}$ borate buffer $\mathrm{pH} 8.5$ for 15 min and then washed with PBS. Blocking was performed in a 3\% bovine serum albumin (BSA), $1 \%$ glycine and $0.3 \%$ Triton-X 100 for $30 \mathrm{~min}$ at room temperature followed by incubation with 1:8000 anti-BrdU monoclonal antibody (Developmental Hybridoma, Iowa City, IA) overnight at $4{ }^{\circ} \mathrm{C}$. Slides were washed with PBS, incubated with 1:1000 Alexa Fluor ${ }^{\circledR} 568$ goat anti-mouse secondary antibody (Molecular Probes) for 1 hour, washed and nuclei were labeled with $1 \mu \mathrm{g} / \mathrm{ml}$ Dapi (Roche) and treated with $2.5 \mathrm{mg} / \mathrm{ml} \mathrm{RNAse} \mathrm{A} \mathrm{at} 37^{\circ} \mathrm{C}$ for 1 hour. Slides were mounted with Moviol mounting solution (Sigma) and BrdU positive cells were counted in a stack of 20 images $(5 \mu \mathrm{m})$ at a Leica laser confocal microscope system (Leica SP2, Wetzlar Gmbh Germany) of the CIGS University of Modena, Italy.

\section{Retinal stem cell differentiation}

In differentiation experiments retinal floating spheres were plated on glass coverslips coated with extracellular matrix (ECM, Sigma). Cells were cultured in DMEM-F12 supplemented with either 20 $\mathrm{ng} / \mathrm{ml} \mathrm{FGF}$ and $2 \mu \mathrm{g} / \mathrm{ml}$ heparin or FGF $+20 \mathrm{ng} / \mathrm{ml}$ PEDF. The cells were allowed to proliferate and migrate out of the sphere over the course of four days. The medium was then replaced with $1 \%$ FBS (Gibco, San Giuliano Milanese, Italy) containing medium and cultured for 15 days.

\section{Immunofluorescence}

Cells grown on glass coverslips were fixed in 4\% paraformaldehyde for 15 min at room temperature. Permeabilization and blocking was performed with $0.2 \%$ TritonX-100 and 3\% bovine serum albumin 
(BSA) (Sigma, St Louis, MO) in PBS for 1 hour followed by incubation with primary antibodies overnight at $4{ }^{\circ} \mathrm{C}$. Cells underwent five washes with PBS and then were incubated with fluorescentconjugated secondary antibodies for 1 hour at room temperature. Primary antibodies used were as follows: 1:1500 anti-Pkc- $\alpha$ rabbit polyclonal (Sigma), 1:100 anti-Pde6b rabbit polyclonal (ABCAM, Cambridge, UK), 1:100 anti-rhodopsin mouse monoclonal 1D4 (Sigma), 1:400 anti-G0 $\alpha$ mouse monoclonal (Chemicon), 1: 750 anti-syntaxin mouse monoclonal (Sigma), 1:1000 anti-calbindin D 28K mouse monoclonal (Sigma). Secondary antibodies were as follows: 1:1000 Oregon Green ${ }^{\circledR} 488$ goat anti-mouse (Molecular Probes, San Giuliano Milanese, and Italy) and 1:1000 Alexa Fluor® 568 goat anti-rabbit (Molecular Probes). Finally slides were mounted in moviol and analyzed using immunofluorescence microscopy Axiocam (Zeiss).

\section{RESULTS}

\section{PEDF promotes Retinal Neurospheres growth and self-renewal.}

Previous studies showed that retinal neurospheres had a limited ability to proliferate and to be expanded in vitro (Giordano et al., 2007, Gu et al., 2006). In all the different conditions retinal neurospheres appeared with a similar morphology and pigmented (Figure 1A). Cells were seeded at low density (40000 cells $/ \mathrm{ml})$ in the growth medium and neurospheres were counted for each growth factor (GF) treatment. As shown in figure 1B, exposure to EGF doubled the floating sphere number compared to the cells plated in the absence of GF, while addictions of FGF increased this number of 3 fold. Moreover, the combination of FGF + EGF enhanced the neurosphere formation of 4 folds compared to the culture without GF. Because PEDF is a neurotrophic factor for some neural population, we set out to test whether the combination of PEDF + FGF enriches the neurosphere population and/or self renewal. The 
addiction of PEDF $(20 \mathrm{ng} / \mathrm{ml})$ to the FGF culture media resulted in a $60 \%$ increase of neurospheres when compared to FGF alone and 20\% more neurospheres than FGF+EGF $(n=3, p<0.01)$.

Our previous studies have shown that EGF alone has a reduced ability to generate secondary neurospheres after passaging while cultures with FGF and EGF+FGF doubled the number of neurospheres at the first cell passage. In order to evaluate whether the positive effect of PEDF was not limited to the primary culture in RSC but could be extended also to RSC self renewal we dissociated single primary generation neurospheres to single cells and re-plated them in the same culture media containing FGF+PEDF. The number of cells capable of generating a secondary neurosphere population increased in the presence of PEDF (figure 1C). We could measure a 5 time increase of passaged neurospheres when exposed to PEDF+FGF compared to FGF alone $(n=4, p<0.001)$. Altogether these data suggest that PEDF favors growth of retinal stem cells in primary cultures and enhances the chances of self-renewal in culture expansions.

\section{Retinal neurosphere proliferation}

A previous study carried on in our lab demonstrated that EGF in the culture medium of RSC favors proliferation during the first days of culture and then proliferation stops. On the other hand, FGF allows high proliferation to be maintained with time in culture. This observation may explain also the positive effect that FGF has on cell passaging. In order to evaluate if the increase in self-renewal properties we observed with PEDF was correlated with enhanced cell proliferation during the neurosphere growth, we labeled retinal spheres with BrdU at the sixth day of the retinal stem cell culture. BrdU positive $\left(\mathrm{BrdU}^{+}\right)$ and BrdU negative $\left(\mathrm{BrdU}^{-}\right)$cells were counted and compared in FGF and FGF+PEDF culture conditions

(figure 2). The presence of PEDF during neurosphere growth promoted cell proliferation more than FGF alone. In particular, FGF gave an average of $20 \%$ of $\mathrm{BrdU}^{+}$cells within each retinal sphere while 
supplementation with PEDF increased this value up to $45 \%$ (Figure 2A). This result indicates that the observed enhanced retinal neurosphere generation is accompanied by increased cell proliferation when RSCs are grown in presence of FGF+PEDF.

\section{Differentiation of retinal cells precursors from RSCs}

The interest in the evaluation of RSC culture treatment correlates with the need of setting the favorable culture conditions to obtain rod photoreceptor differentiation. Several studies have indicated the possibility of using RSCs as a donor source. We investigated the ability of differentiated RSCs to express typical markers of adult retinal cells. In our previous work we have evaluated the effect of different GF combinations on retinal cell differentiation. After 6 days of growth, retinal spheres were plated onto an extracellular matrix substrate for 4 days to allow progenitor cells to exit, attach and start differentiation (Figure 3). After 4 days the culture medium was replaced with media containing $1 \%$ FBS. Retinal progenitor cells (RPCs) were cultured up to 20 days and then underwent expression analysis by immunofluorescence labeling. Rod photoreceptor-like cells were defined by the co-expression of specific rod markers: Rhodopsin and Pde6b (Figure 4 B). Rod-like cells were also characterized by smaller cell size accompanied by smaller nuclei compared to the Rho/Pde6b negative cells. Bipolar-like cells were identified by co-expression of $\mathrm{G}_{0} \alpha$ and Pkc $\alpha$ (fig.4 C). Our culture condition was also favorable for expression of horizontal and amacrine markers like calbindin and syntaxin respectively (data not shown). Generation of neurospheres in the presence of FGF+PEDF did not significantly alter the number of rod-like cells that reached 30 to $40 \%$ of the total cultured cells (Fig.4 A). In turn, we were not able to detect bipolar-like cells in the FGF+PEDF culture while 5\% of cells treated with FGF only were positive to $\mathrm{G}_{0} \alpha / \mathrm{Pkc} \alpha$. 
Altogether, results on retinal cell differentiation indicate that PEDF does not significantly affect rod-like differentiation.

\section{Discussion}

Few cells in the mammalian adult ciliary body have the ability to proliferate when subjected to the appropriate stimuli (Tropepe et al., 2000; Ahmad et al., 2000; Zhao et al., 2002). This capability, which resides in less than $1 \%$ of the ciliary body cells, can be ameliorated with exposure to growth factors in the culture media. In our previous work we have demonstrated that addiction of FGF + EGF gave the highest neurosphere yield and that this property is maintained also over daughter neurosphere generation after sphere passage (Giordano et al., 2007). Despite the enhanced neurosphere yield, the presence of EGF decreased the rod-like cell number during RSC differentiation and increased the bipolar-like marker expression. This latter result prompted us to investigate alternative GF combinations in order to maximize the RSC self-renewal without affecting the photoreceptor-like cell amount during the following differentiation. Here we report that PEDF in combination with FGF ameliorates the neurosphere formation, likewise acting on the self-renewal capability of the RSCs. This property already described by PEDF on NSC derived from the adult SVZ (Ramirez-Castillejo et al., 2006), was also confirmed during retinal neurosphere passaging. Self-renewal involves both proliferation and maintenance of undifferentiated state, although this process is still poorly understood at molecular levels. We propose here that PEDF might be a modulator during cell division likely promoting generation of two identical stem cells or one stem cell and one committed progenitor instead of two committed progenitor cells. Unlike NSC, (Ramirez-Castillejo et al., 2006) we find that PEDF has a mitogen effect on RSC as indicated from BrdU incorporation during neurosphere growth. This might be 
due to cell-intrinsic mechanisms (Arsenijevic 2003) or due to the lack of some external cues such as growth promoting NSC culture conditions.

Upon differentiation, PEDF does not interfere with rod-like cell marker expression giving a similar amount of $\sim 35 \%$ to those cells differentiated in the FGF only medium. However, the true identity of photoreceptor-like cells with further molecular and functional analysis remains to be investigated. Although PEDF has no influence on the rod-like cells number, it causes a lack in bipolar-like cell amount. This effect suggests a possible role of PEDF in Chx 10 gene regulation since it has been reported in the involvement of this transcription factor in bipolar cell differentiation (Livne-Bar et al., 2006; Kokkinopoulos et al., 2008). The fact that loss of bipolar-like cells is not accompanied by an increase in other interneuron or rod-like cells may lead to enrichment in progenitor cells still capable of being addressed toward a diverse retinal cell fate, however, this needs to be documented by further studies.

Taken together, these findings indicate that PEDF may contribute to ameliorate RSC expansion, offering a source of alternative therapy in regenerative medicine.

\section{Acknowledgements}

We are grateful to Fondazione Cassa di Risparmio di Modena for Dr De Marzo fellowship support and XIII International Symposium on Retinal Degeneration for her travel award grant. We acknowledge the CIGS of University of Modena for providing confocal microscopy assistance. This work was supported by research grants EVI-GENORET: LSHG-CT-2005-512036 from the European Community, by research grant GGP06096 from Fondazione Telethon and PRIN 2006053302_003.

\section{Bibliography}


Ahmad I, Tang L, Pham H. (2000). Biochem Biophys Res Commun; 270:517-521

Arsenijevic Y. (2003). Mol Neurobiol.; Feb;27(1):73-98

Becerra SP, Sagasti A, Spinella P et al. (1995) J Biol Chem; 270: 25992-25999

Coles BL, Angenieux B, Inoue T et al. (2004) Proc Natl Acad Sci USA;101:15772-15777

Giordano F, De Marzo A, Vetrini F and Marigo V (2007). Mol Vis.; 13:1842-1850

Gu P, Harwood LG, Zhang X et al.,(2007) Mol Vis.;13:1045-57.

Inoue Y, Yanagi Y, Tamaki Y et al. (2005) Exp Eye Res; Oct; 81(4) :437-45

Moshiri A, Close J, Reh TA. (2004) J Dev Biol.;48(8-9):1003-14

Nishiguchi KM, Kaneko H, Nakamura M et al., (2008). Invest Ophthalmol Vis Sci. Jan;49(1):422-8.

Pignolo RY, Francis MK, Rotemberg MO et al. (2003) J Cell Physiol. 195(1): 12-20

Ramirez-Castillejo C, Sanchez-Sanchez F, Andreu-Agullo C et al. (2006) Nat Neurosci; 9(3):331-338

Tropepe V, Coles BL, Chiasson BJ et al.(2000) Science;287 :2032-2036.

Tombran-Tink J, Chader GG, \& Johnson LV. (1991) Exp Eye Res. 53:411-414 


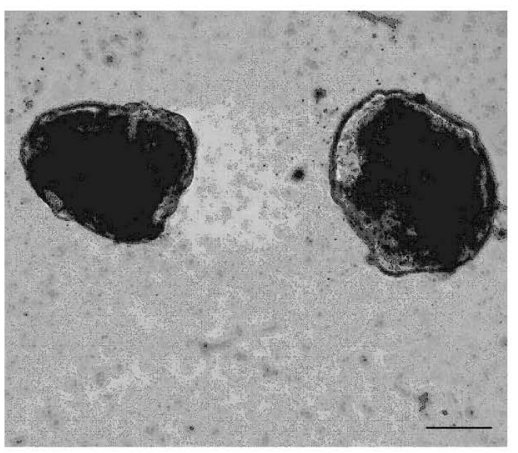

C

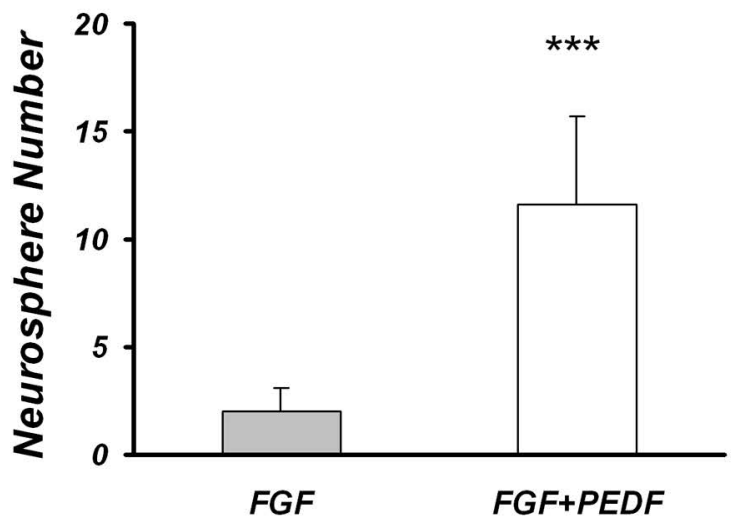

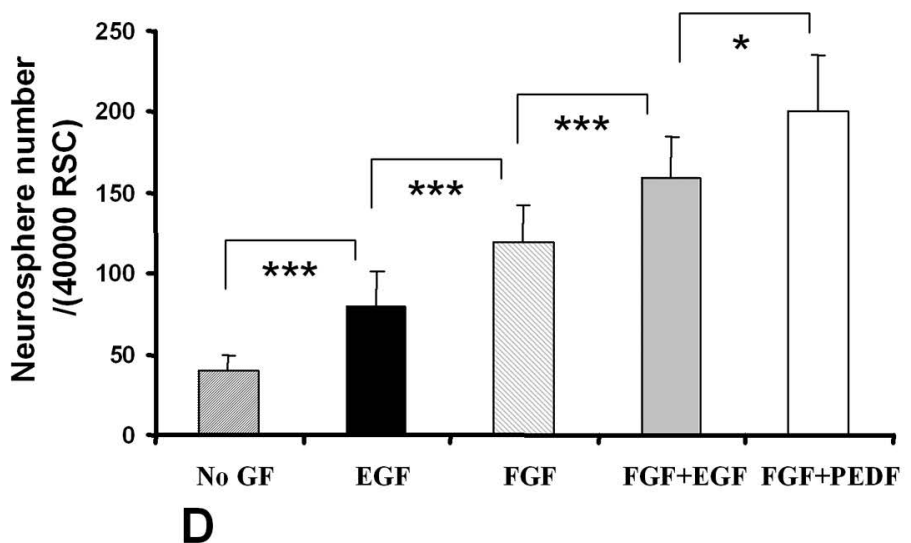

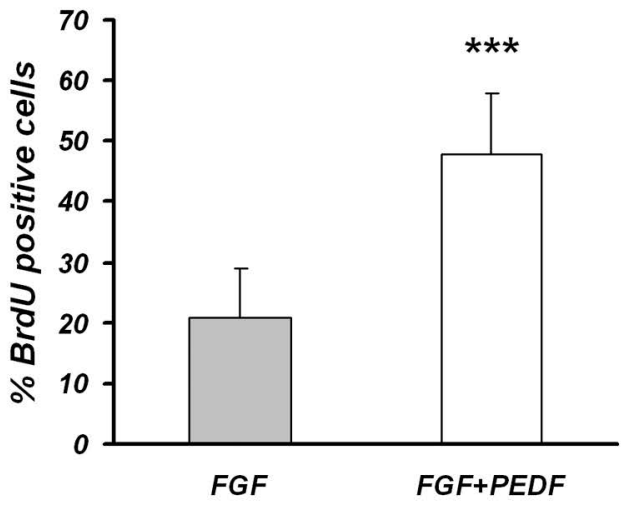


A
$\mathrm{SFM}+\mathrm{GF}$
$1 \%$ FBS
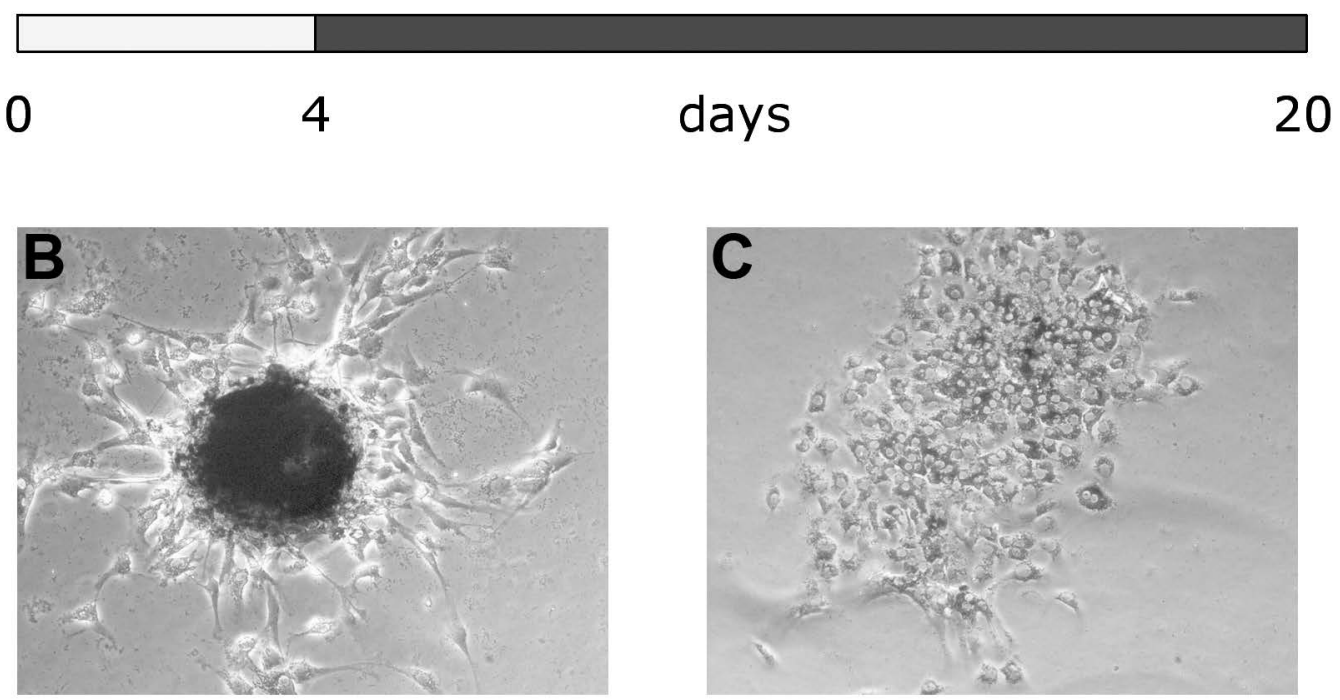
A

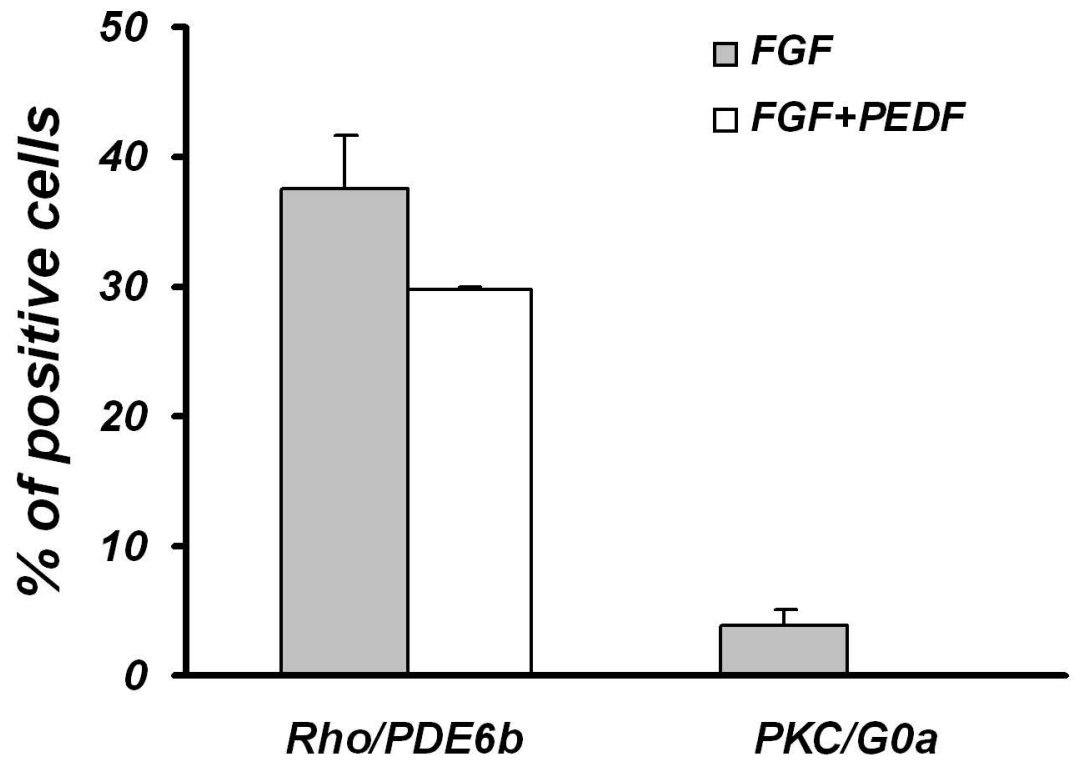

B

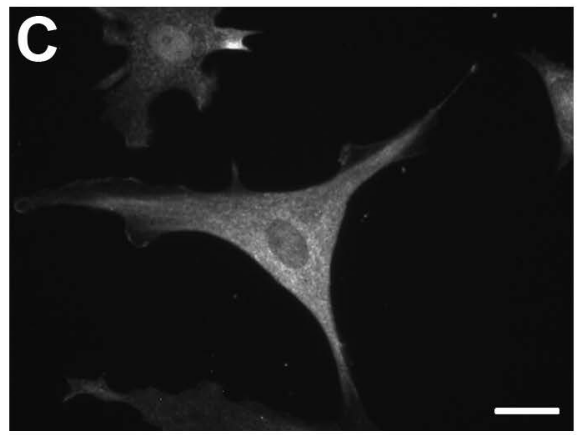

